\title{
Programming Hydrogen Production via Controllable Emulsification/Demulsification in a Switchable Oil-Water System
}

Yuchen Zhang, ${ }^{1}$ Haomiao Zhang, ${ }^{1}$ Pingwei Liu, ${ }^{1,2, *}$ Hailong Sun, ${ }^{3, *}$ Bo-Geng Li, $^{1}$ and Wen-Jun Wang $^{1,2, *}$

${ }^{1}$ State Key Laboratory of Chemical Engineering, College of Chemical and Biological Engineering, Zhejiang University, 38 Zheda Road, Hangzhou, China 310027

${ }^{2}$ Institute of Zhejiang University - Quzhou, 78 Jiuhua Boulevard North, Quzhou, China 324000

${ }^{3}$ State Key Laboratory of Hydraulics and Mountain River Engineering, Sichuan University, 24 South Section 1, Yihuan Road, Chengdu, China 610064

* Email address of corresponding authors: liupingwei@zju.edu.cn (P.W. Liu); sunhl@ssu.edu.cn (H.L. Sun); wenjunwang@zju.edu.cn (W.-J. Wang)

The supporting information contains 20 pages including detailed experimental parts, Figure S1 to S15 and Table S1 to S3. 


\section{Part 1. Stimuli-responisve microgel-stablized emulsion}

\subsection{Stability and switchability}

1.2 Interfacial area of droplets

\section{Part 2. Experimental parts}

2.1 Synthesis of PVP-Au hybrid

2.2 Synthesis 1-dodecanethiol stabilized AuNPs

2.3 Dynamic emulsion catalysis via oscillating $\mathrm{CO}_{2} /$ vacuum signal to boost the catalysis

2.4 Activities comparison of different systems

\section{Part 3. Figures and tables}

Figure S1. Size distribution of MG3 without loading metal NPs from TEM results.

Figure S2. ${ }^{1} H$ NMR spectra of monomer residues in aqueous MG1-3 samples.

Figure S3. Size increasse upon $\mathrm{CO}_{2}$ purging of different MGs

Figure S4. Photos of Pickering emulsion

Figure S5. Volume fraction of n-dodecane/aqueous biphasic systems at different stages

Figure S6. $\mathrm{CO}_{2}$ solubility in water and their corresponding $\mathrm{pH}$; high pressure $\mathrm{CO}_{2}$ induced demusification of a Pickering emulsion

Figure S7. Volume fraction of oil stayed in emulsion phase and water

Figure S8. Size distribution and TEM images of MG3 supported AuNPs and PdNPs

Figure S9. Photos of Pickering emulsion after 0, 1, 3, 10, 30min $\mathrm{CO}_{2}$ bubbling of MG3 and MG3-Au

Figure S10. Volume fraction of free oil and water of MG3, MG3-Au and MG3-Pd after bubbling $\mathrm{CO}_{2}$ for $30 \mathrm{~min}$. 
Figure S11. Histogram of activity in three different systems

Figure S12. ${ }^{1} \mathrm{H}$ NMR spectra of MG3-Au catalyzed dimethylphenylsilane oxidation at different reaction times.

Figure S13. GC traces of generated $\mathrm{H}_{2}$.

Figure S14. Microscope images and photos of MG3-Au stabilized Pickering emulsion

Figure S15. ${ }^{1} \mathrm{H}$ NMR spectra of dimethylphenylsilane emulsion prior and subsequent to aging for one week at room temperature.

Table S1. Conversion in different systems with/without $\mathrm{CO}_{2}$ treatment

Table S2. Conversion of silane oxidation of MG3-Au and recycles

Table S3. Conversion of olefin hydrogenation on MG3-Pd and recycles 


\section{Stimuli-responisve microgel-stablized emulsion}

\subsection{Stability and switchability}

We first study from the point of desorption work vs. chemical potential by considering the MG particles as compact spherical particles adsorbed at interfaces as with a typical Pickering emulsion. The work required to remove a spherical particle of radius $r$ from interface to water phase is given by $^{1}$

$$
W^{\text {desorption }}=\pi r^{2} \gamma_{0}(1-\cos \theta)^{2}
$$

where $\gamma_{0}$ is interfacial tension between oil and water. Assuming $f_{w}$ and $f_{s}$ are the fractions of MG particles in the water and on the surfaces, respectively, and $f_{w}+f_{s}=1 ; N_{p}$ is the total number of particles in a water volume of $V_{w}$. The chemical potential of a particle in the water will be ${ }^{2}$

$$
\mu_{w}^{2}=\mu_{w}^{0}+4 \pi r^{2} \gamma_{w s}+k T \ln \left(N_{p} f_{w} a_{w} / V_{w}\right)
$$

where $\gamma_{w s}$ is interfacial tension between water and spherical particle; $\mu_{w}^{0}$ is a reference chemical potential and $a_{w}$ is a suitable activity coefficient. After bubbling $\mathrm{CO}_{2}$ in the system the chemical potential change can be written as

$$
\mu_{w}^{1}-\mu_{w}^{2}=4 \pi r_{1}^{2} \gamma_{1, w s}-4 \pi r_{2}^{2} \gamma_{2, w s}+k T \ln \left(f_{1, w} / f_{2, w}\right)
$$

If $\mu_{w}^{1}-\mu_{w}^{2}>W^{\text {desorption }}$, then the microgels will spontaneously transfer to aqueous media upon purging $\mathrm{CO}_{2}$ and the emulsion breaks. The key parameter is interfacial tension $\gamma_{w s}$ variation before and after introducing $\mathrm{CO}_{2}$, which is mainly determined by chemistry and composition of the microgel particles.

Meanwhile, microgels-stabilized emulsions also show some non-Pickering emulsion properties. In particular, microgels can be strongly swollen and deformed at oil-water interface to cover more area thus stabilizing oil phase..$^{3-5}$ For this aspect, cross-linking density is the key factor which determines 
the capability of deformation. Microgels with a lower cross-linking density $D_{c}$ have a looser structure with a better capability of deformation for emulsion stabilization. ${ }^{3}$ Upon bubbling $\mathrm{CO}_{2}$, these microgels are more swollen and deforming which has positive effects on stabilization. At the same time, these MGs have a higher $\mathrm{CO}_{2}$ uptake efficiency and better switching performance, which has negative effects on stabilization as discussed above. For this $\mathrm{CO}_{2}$-responsive stabilized Pickering emulsion, MGs detachment from interfaces and deformation at interfaces would both contribute to the demulsification process. We can estimate that better hydrophilicity $(h)$ (mainly controlled by the chemistry and composition of the microgel particles), and a proper degree of cross-linking $\left(D_{c}\right)$ are helpful in demulsification. Also, a higher $\mathrm{CO}_{2}$ concentration during the process would induce a quicker and more complete demulsification.

\subsection{Interfacial area of droplets}

For a phase boundary system, we assume the droplet size is about $400 \mu \mathrm{m}$ under stirring, which is somewhat smaller than its real state. The total surface would be $3 \times 10^{-4} \mathrm{~m}^{2}$ in phase boundary system. By measuring the size of all droplets in Figure 4c, we can estimate the total oil volume to be about $7.66 \times 10^{-6} \mathrm{~mL}$ with a surface area of about $8.8 \times 10^{-3} \mathrm{~cm}^{2}$. So for $2 \mathrm{~mL}$ of oil phase, the total surface area is about $0.23 \mathrm{~m}^{2}$ in Pickering emulsion system, which is about three orders of magnitude higher than phase boundary system. Such a large surface area in Pickering emulsion with metal nanocatalytsts located at surface ensures a high activity. 


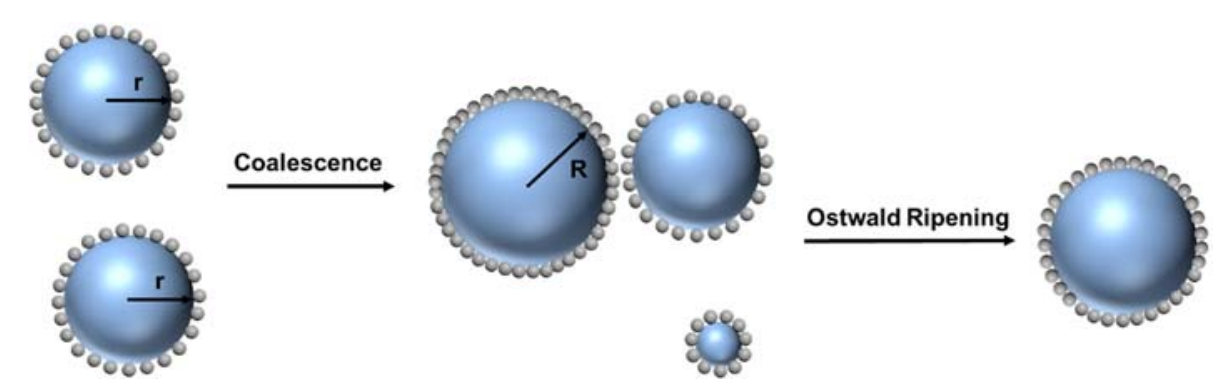

Scheme S1. Scheme of coalescence and ripening in Pickering emulsion.

We caculate the decrease in interfacial area due to coalescence using the merging of two identical droplets. $V_{\text {total }}$ is the total volume of these two droplets with a radius of $r$ which remains unchanged after coalescence to form a bigger droplet with a radius of $R$. Then we got

$$
\mathrm{V}_{\text {total }}=\frac{4}{3} \pi r^{3} \times 2=\frac{4}{3} \pi R^{3}
$$

The initial interfacial area $a$ and final area $A$ of the bigger droplet can then be easily caculated as

$$
A=4 \pi R^{2} \approx 6.35 \pi r^{2} \approx 0.8 a
$$

Then the decrease of such a process approximates $20 \%$ and about a $26 \%$ increase of packing density would arise. The calculation can be extended to all ripening and coalescence processes in Pickering emulsions, which would decrease the interfacial area. 


\section{Experimental}

\subsection{Synthesis of PVP-Au hybrid}

The synthesis procedure for the PVP-Au hybrid was followed as described previously. ${ }^{6}$ Specifically, $333.3 \mathrm{mg}$ of PVP was added to an aqueous solution of $\mathrm{HAuCl}_{4}(1 \mathrm{mM}, 30 \mathrm{~mL})$ so that the molar ratio of $\mathrm{AuCl}_{4}{ }^{-}$and monomer unit of PVP was kept at 1:100. A K-30 PVP having average molecular weight of $40 \mathrm{kDa}$ was used. The mixture was further stirred for $30 \mathrm{~min}$ in an ice bath. Then, an aqueous solution of $\mathrm{NaBH}_{4}(100 \mathrm{mM}, 3 \mathrm{~mL})$ was rapidly sprayed into the mixture under vigorous stirring. The color of the mixture immediately turned from pale yellow to dark brown, indicating the formation of small Au NPs. The PVP-Au hybrids were subsequently dialyzed overnight to remove inorganic impurities.

\subsection{Synthesis 1-dodecanethiol stabilized AuNPs}

The synthesis procedure for 1-dodecanethiol stabilized AuNPs was followed as described previously. ${ }^{7}$ An aqueous solution of $\mathrm{HAuCl}_{4}\left(15 \mathrm{~mL}, 30 \mathrm{mmol} / \mathrm{dm}^{3}\right)$ was mixed with a solution of tetraoctylammonium bromide in toluene $\left(40 \mathrm{~mL}, 50 \mathrm{mmol} / \mathrm{dm}^{3}\right)$. The two-phase mixture was vigorously stirred until all the tetrachloroaurate was transferred to the organic phase. Dodecanethiol (85 mg) was then added to the organic phase. A freshly prepared aqueous solution of sodium borohydride $\left(25 \mathrm{~mL}, 0.4 \mathrm{~mol} / \mathrm{dm}^{3}\right)$ was slowly added with vigorous stirring. After further stirring for $3 \mathrm{~h}$, the organic phase was separated, evaporated to $6 \mathrm{~mL}$ in a rotary evaporator and mixed with 200 $\mathrm{mL}$ ethanol to remove excess thiol. The mixture was kept for $4 \mathrm{~h}$ at $-18^{\circ} \mathrm{C}$ and the dark brown precipitate was filtered off and washed with ethanol. 


\subsection{Dynamic emulsion catalysis via oscillating $\mathrm{CO}_{2} /$ vacuum signal to boost the catalysis}

For MG-Au system, $2 \mathrm{~mL}$ silanes were introduced into $5 \mathrm{~mL}$ catalyst aqueous solution. The formed two-phase system was homogenized at 10,000 rpm for $1 \mathrm{~min}$. The mixture was then stirred at 750 rpm with a magnetic stir rod at $25{ }^{\circ} \mathrm{C}$ for a given reaction time. For another two sets, the system was vacuumized/pressurized with $\mathrm{CO}_{2}$ every one or two hours. Conversions were determined by ${ }^{1} \mathrm{H} \mathrm{NMR}$.

For the PVP/1-dodecanethiol system, $2 \mathrm{~mL}$ silanes were introduced into the $5 \mathrm{~mL}$ catalyst aqueous solution. The formed two-phase systems were homogenized at 10,000 rpm for $1 \mathrm{~min}$ and then stirred at $750 \mathrm{rpm}$ with a magnetic stir rod at $25{ }^{\circ} \mathrm{C}$ for a given reaction time. The system was vacuumized/pressurized with $\mathrm{CO}_{2}$ every 24 hours. In every cycle, conversions were determined by ${ }^{1} \mathrm{H}$ NMR every $8 \mathrm{hr}$.

\subsection{Activities comparison of different systems}

Reaction conditions: Triethylsilane $(5 \mathrm{mmol})$, water $(5 \mathrm{~mL})$, in Pickering emulsion and phase boundary systems; Triethylsilane $(1 \mathrm{mmol})$, water $(1 \mathrm{~mL})$, ethanol $(4 \mathrm{~mL})$ in organic solution. AuNPs/triethylsilane $=0.5 \mathrm{~mol} \%, 25^{\circ} \mathrm{C}, 1 \mathrm{~h}$. 


\section{Figures and tables}
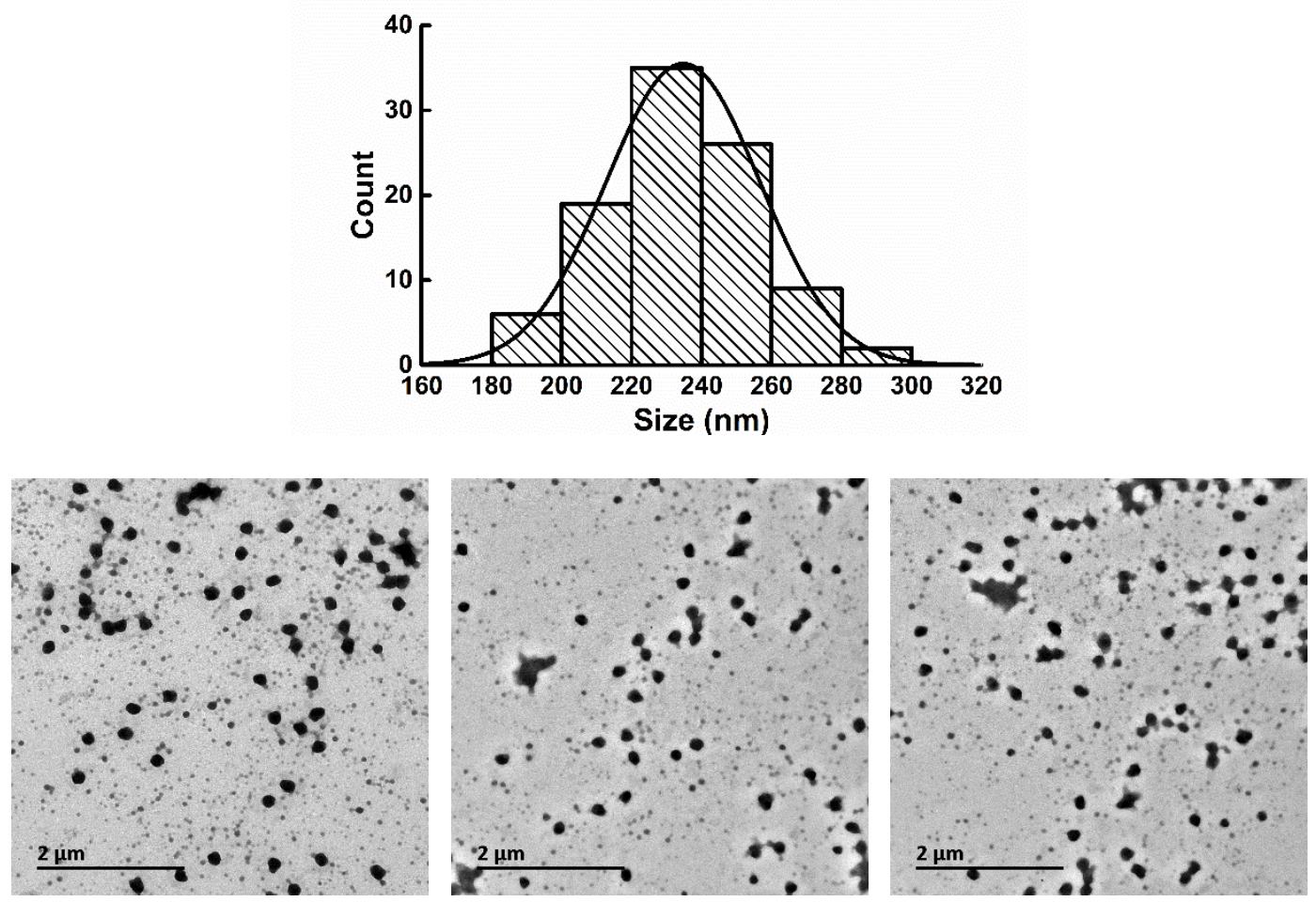

Figure S1. Size distribution of MG3 without loading metal NPs from TEM results. Samples were stained using uranyl acetate. 

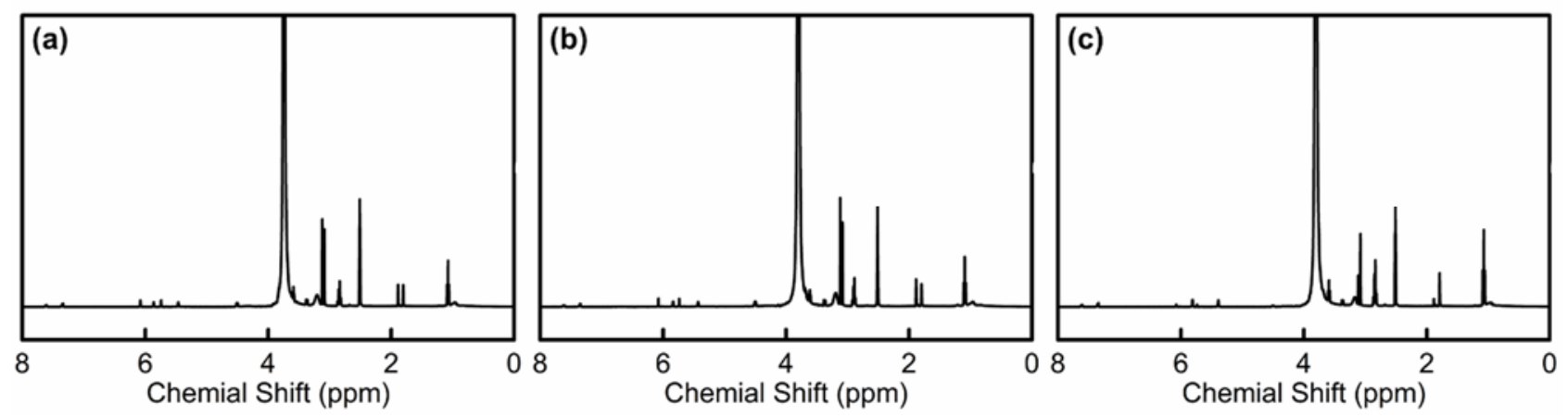

Figure S2. ${ }^{1} \mathrm{H}$ NMR spectra of monomer residues in aqueous MG1-3 samples using benzoic acid as an internal standard. a) MG1, b) MG2, and c) MG3.

After polymerization, $2 \mathrm{~mL}$ of aqueous MG sample was filtrated using a syringe with $0.45 \mu \mathrm{m}$ filter membrane. Benzoic acid $(1.2 \mathrm{mg})$ was then added into $0.2 \mathrm{~g}$ of the filtrate. DMSO-d6 was used as solvent for the NMR measurement of the filtrate. The amount of unreacted monomers in the aqueous MG sample were determined with benzoic acid as an internal standard, by comparing peak areas of benzoic acid $(\delta=7.35$ and $7.62 \mathrm{ppm})$ with those of double bounds in monomers $(\delta=5.4$ to $6.1 \mathrm{ppm})$ and methylenes at tertiary amine group of DEAEMA ( $\delta=2.85$ to $2.95 \mathrm{ppm})$. The monomer conversions and compositions in the MG were thus estimated. 
(a)

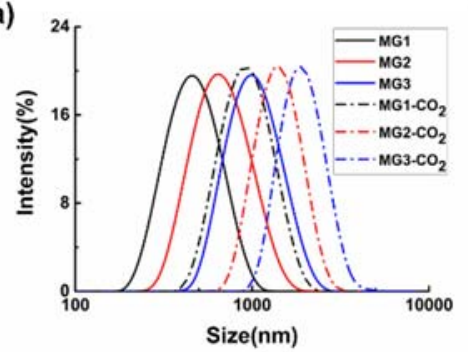

(b)

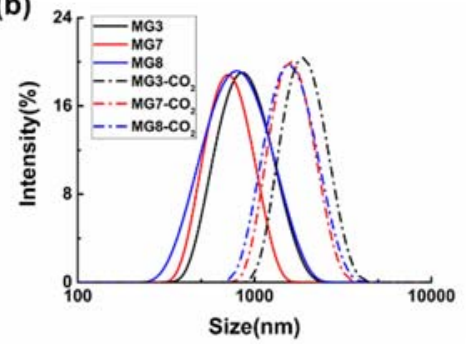

(c)

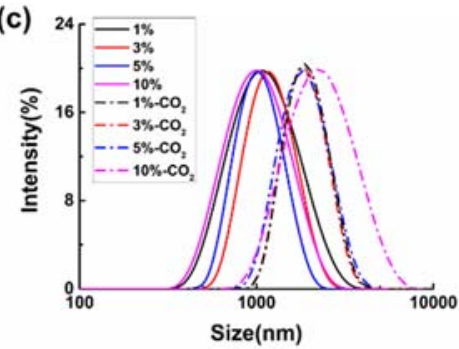

Figure S3. Size increase upon $\mathrm{CO}_{2}$ purging of different MGs.

a

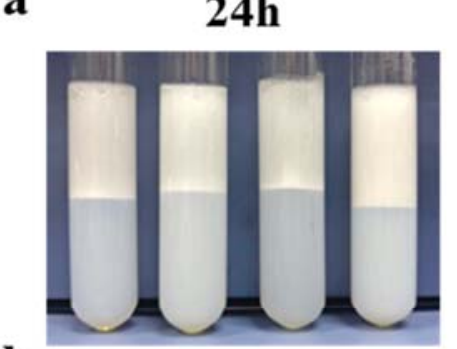

b

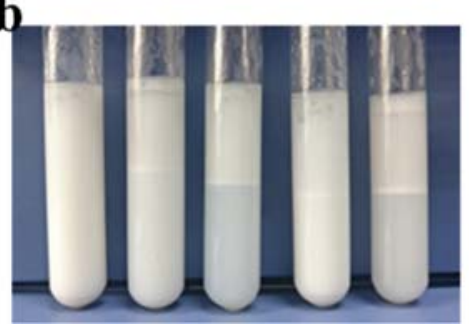

$3 \mathrm{~min} \mathrm{CO}_{2}$
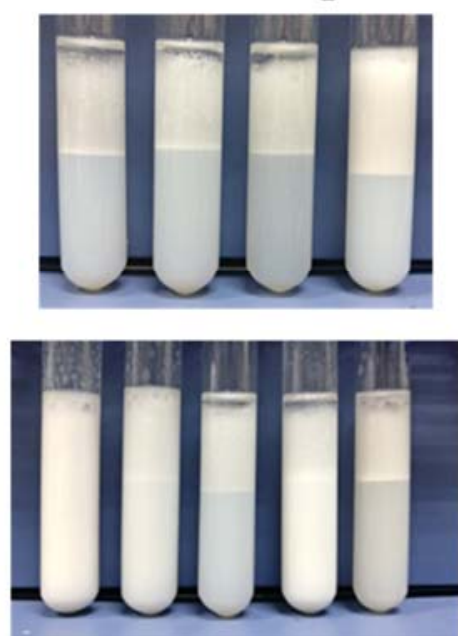

$30 \mathrm{~min} \mathrm{CO}_{2}$
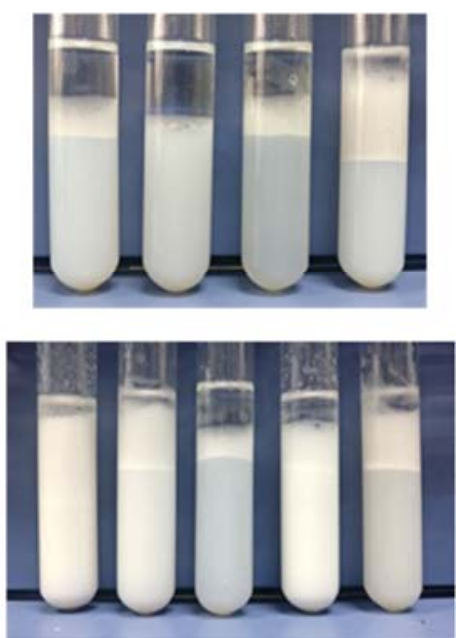

Figure S4. a) Photos of Pickering emulsion stabilized by MG4, MG3, MG5, MG6 (from left to right); b) Photos of Pickering emulsion stabilized by MG8, MG7, MG3, MG1, MG2 (from left to right). 


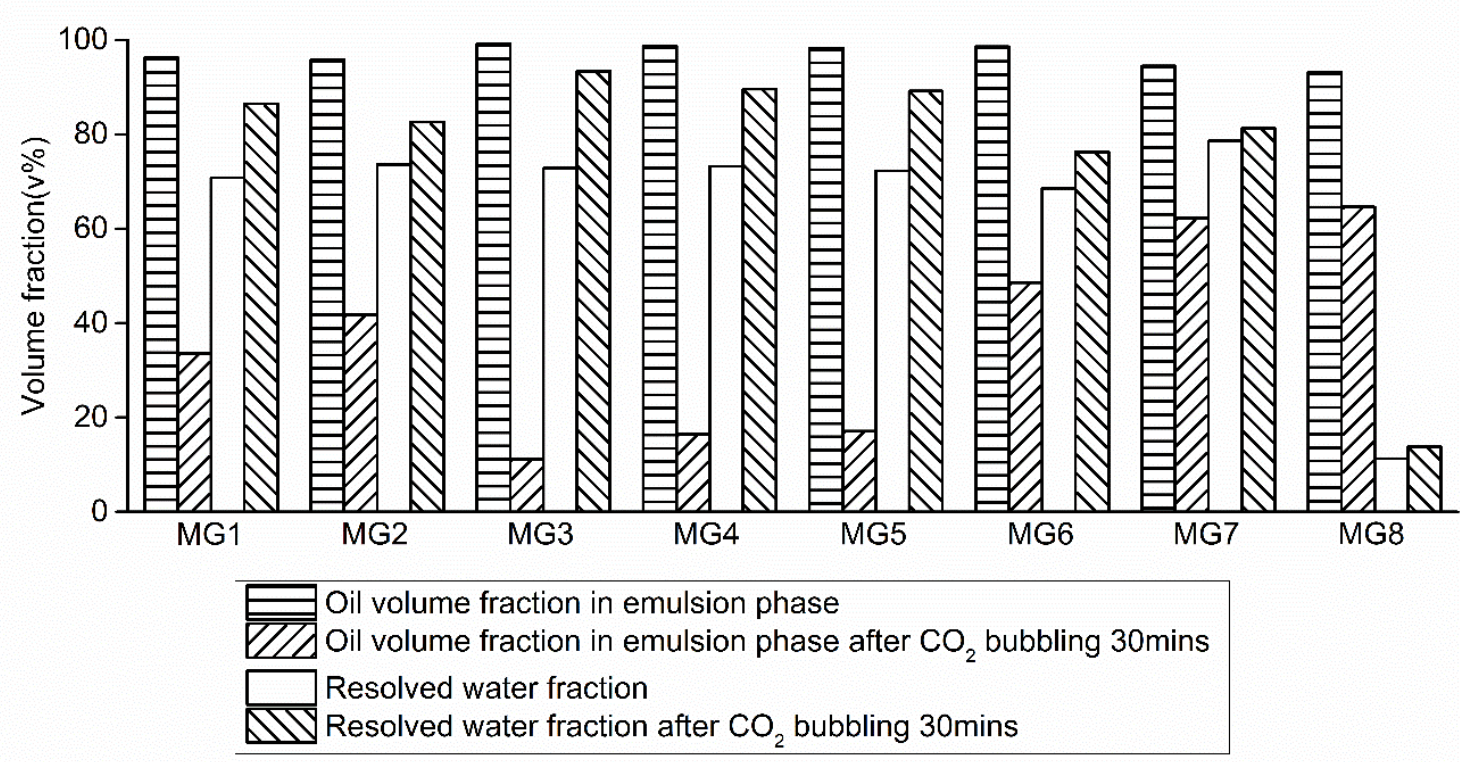

Figure S5. Volume fraction of n-dodecane / aqueous biphasic systems at different stages.
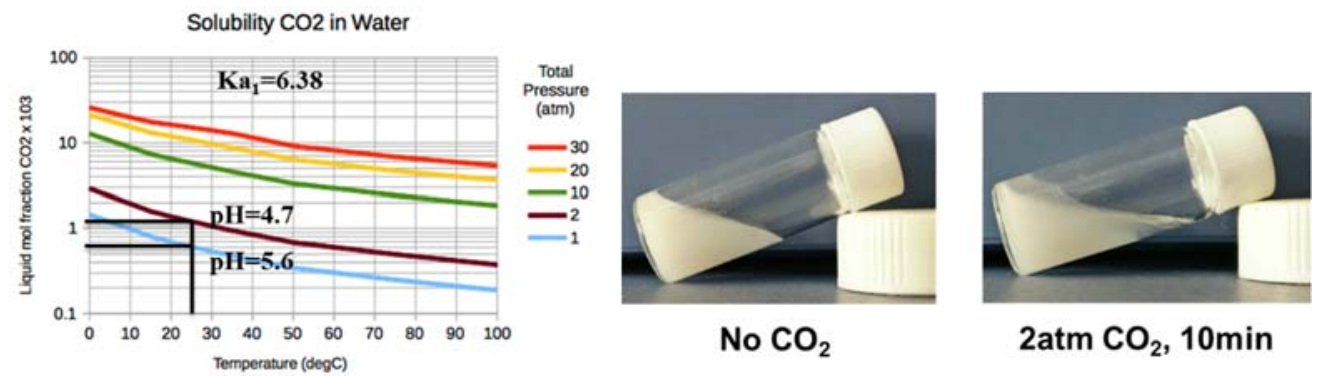

Figure S6. $\mathrm{CO}_{2}$ solubility in water and the corresponding $\mathrm{pH}$; high pressure $\mathrm{CO}_{2}$ induced demusification of a Pickering emulsion.

Since $\mathrm{CO}_{2}$-swithability is $\mathrm{pH}$-switchability in nature, we assume high pressure $\mathrm{CO}_{2}$ could induce even quicker demusification in Pickering emulsions. As shown in Figure S4, $\mathrm{pH}$ can drop to 4.7 under 2 atm $\mathrm{CO}_{2}$ under ideal conditions. The actual $\mathrm{pH}$ in our experiments was about 5.3 indicating $94 \%$ protonation of DEAEMA units. Thus quicker and more complete separation of oil and aqueous phase was observed. Similarly, vacuum can also replace $\mathrm{N}_{2}$ treatment to reset MGs to original state with shorter time. 

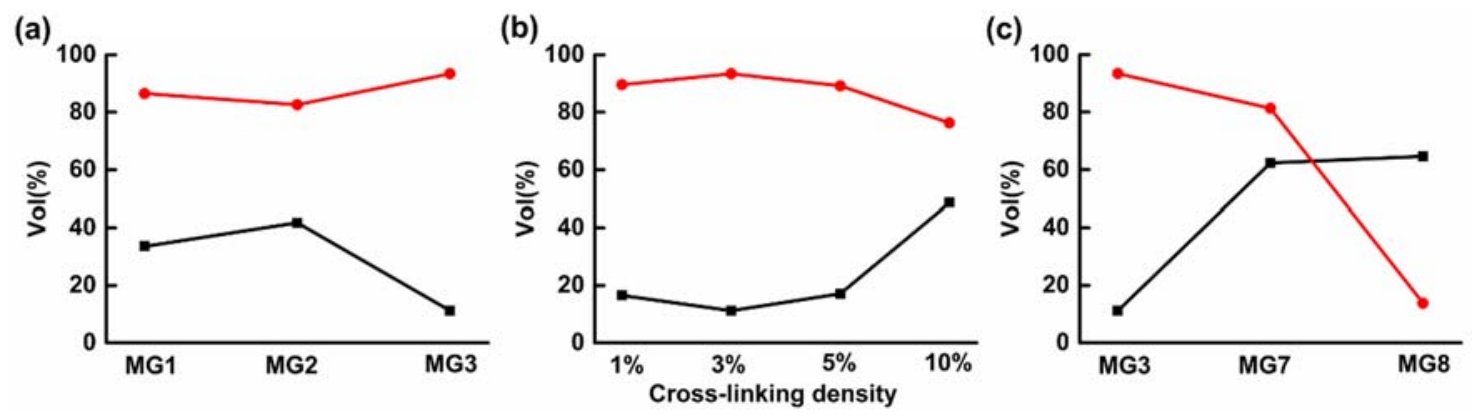

Figure S7. Volume fraction of oil stayed in emulsion phase (black) and water (red) after bubbling $\mathrm{CO}_{2}$ for 30 min. a) Comparison of MGs with different ionic monomer type; b) comparison of MGs of different cross-linking density; c) comparison of MGs of different monomer ratios.
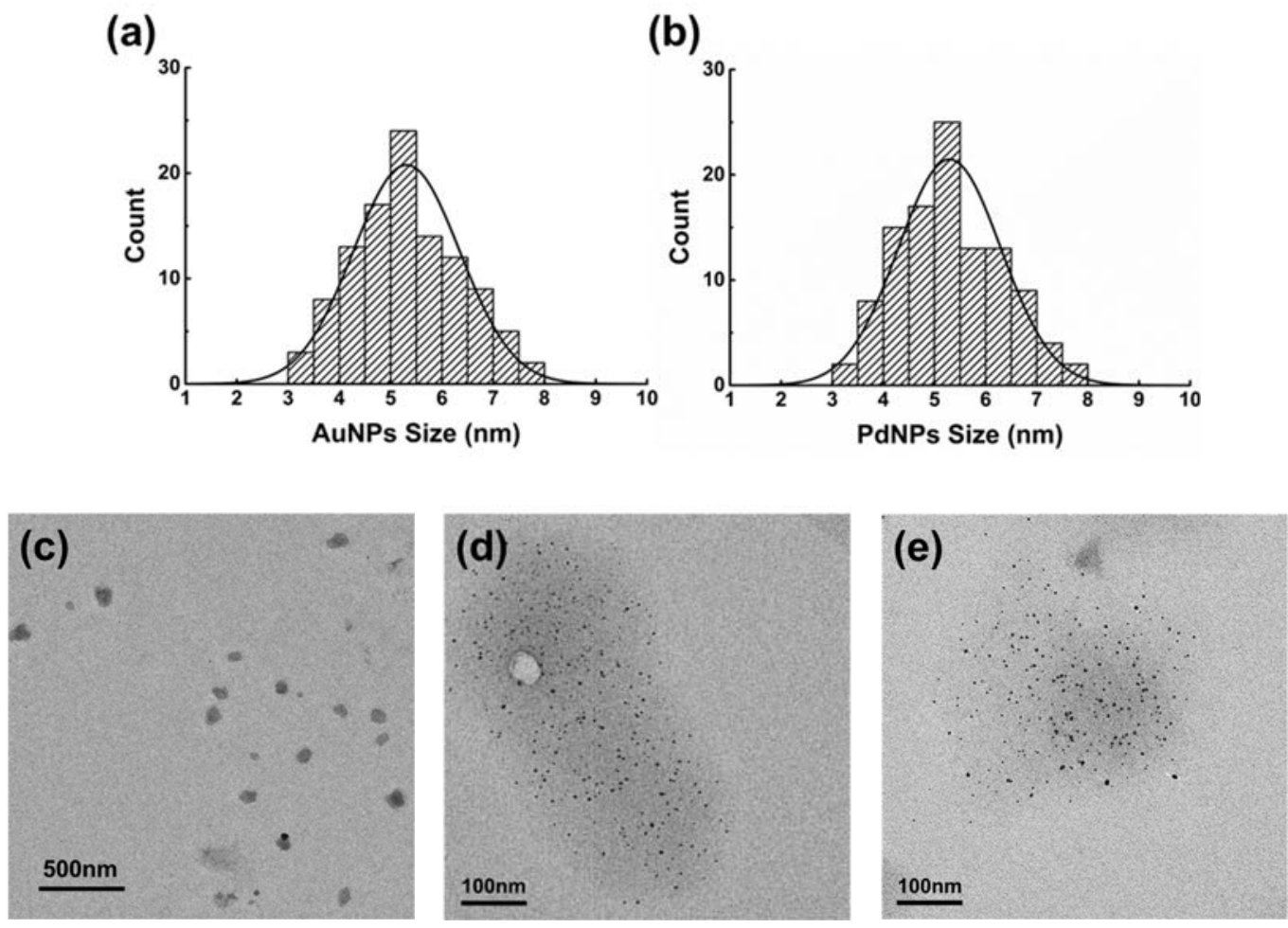

Figure S8. Size distribution and TEM images of MG3 supported AuNPs (a, c, and d) and MG3 supported PdNPs (b and e). 


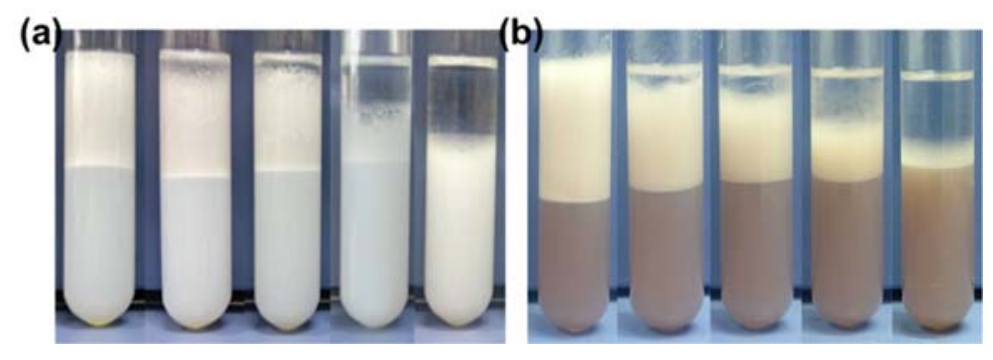

Figure S9. Photos of Pickering emulsion after 0, 1, 3, 10, 30 min. (left to right) $\mathrm{CO}_{2}$ bubbling of (a) MG3 and (b)MG3-Au.
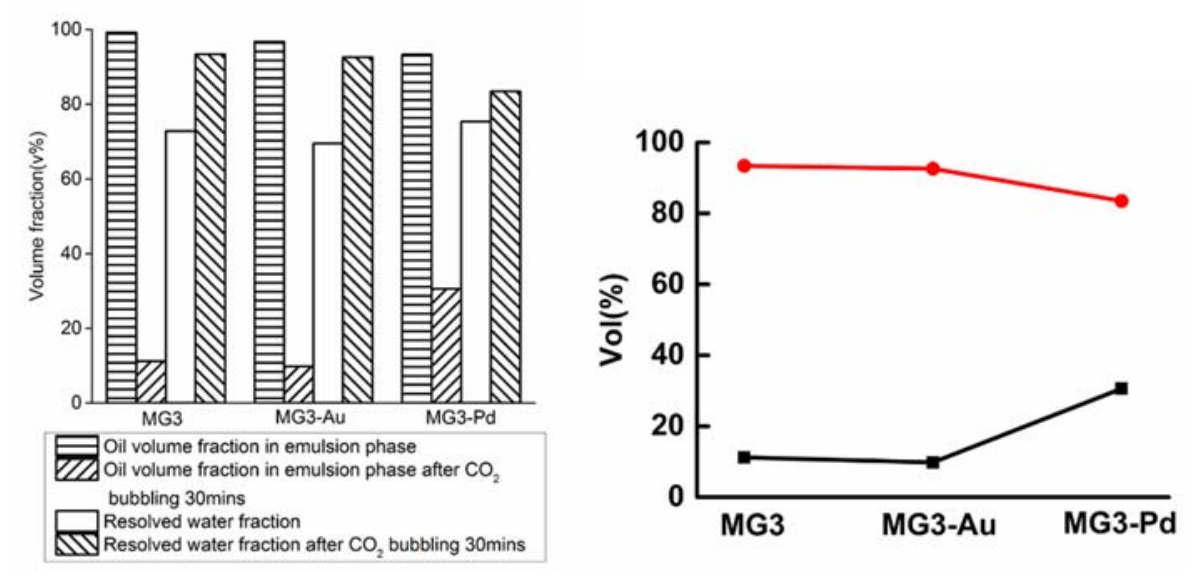

Figure S10. Volume fraction of free oil (black) and water (red) of MG3, MG3-Au and MG3-Pd after bubbling $\mathrm{CO}_{2}$ for $30 \mathrm{~min}$.

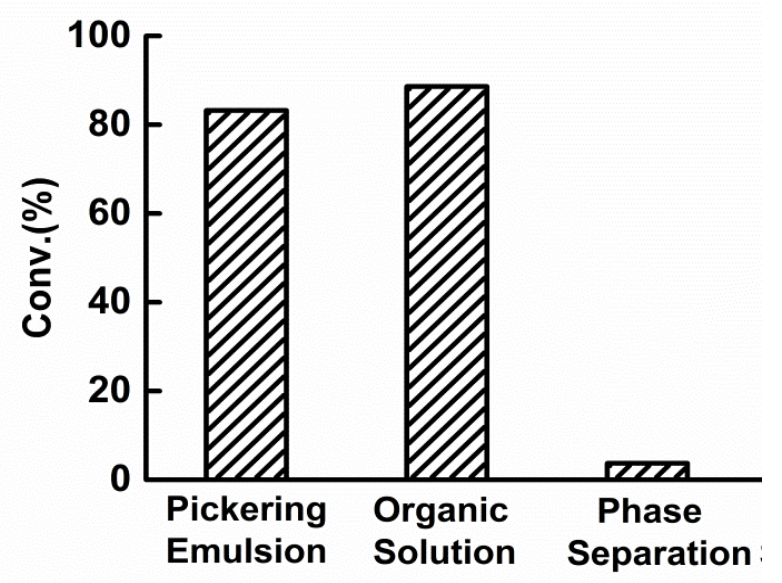

Figure S11. Histogram of activity in the three systems. 


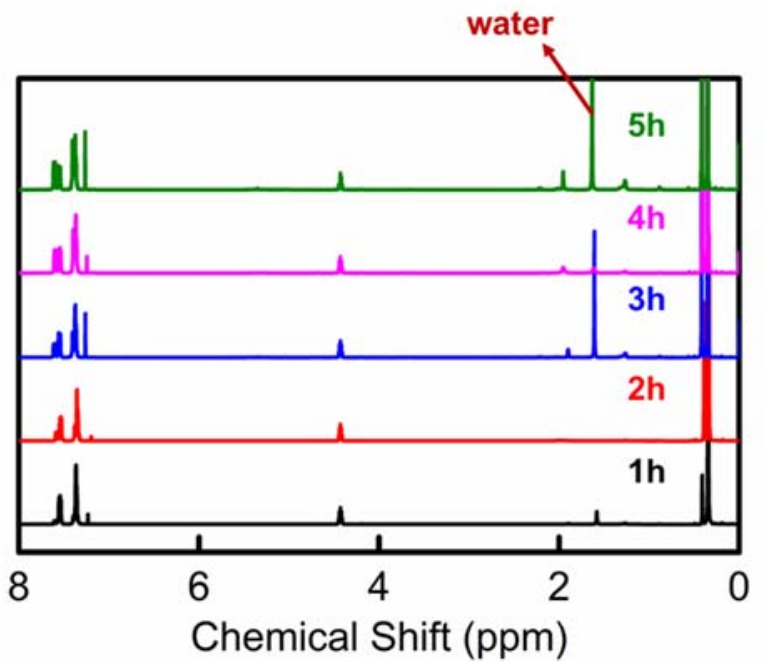

Figure S12. ${ }^{1} \mathrm{H}$ NMR spectra of MG3-Au catalyzed dimethylphenylsilane oxidation at different reaction times. Dimethylphenylsilane (2 mL) was introduced into $6 \mathrm{~mL}$ of MG3-Au aqueous solution, at a ratio of AuNPs to dimethylphenylsilane of $0.05 \mathrm{~mol} \%$. The reaction was conducted at $25^{\circ} \mathrm{C}$ and $750 \mathrm{rpm}$. 

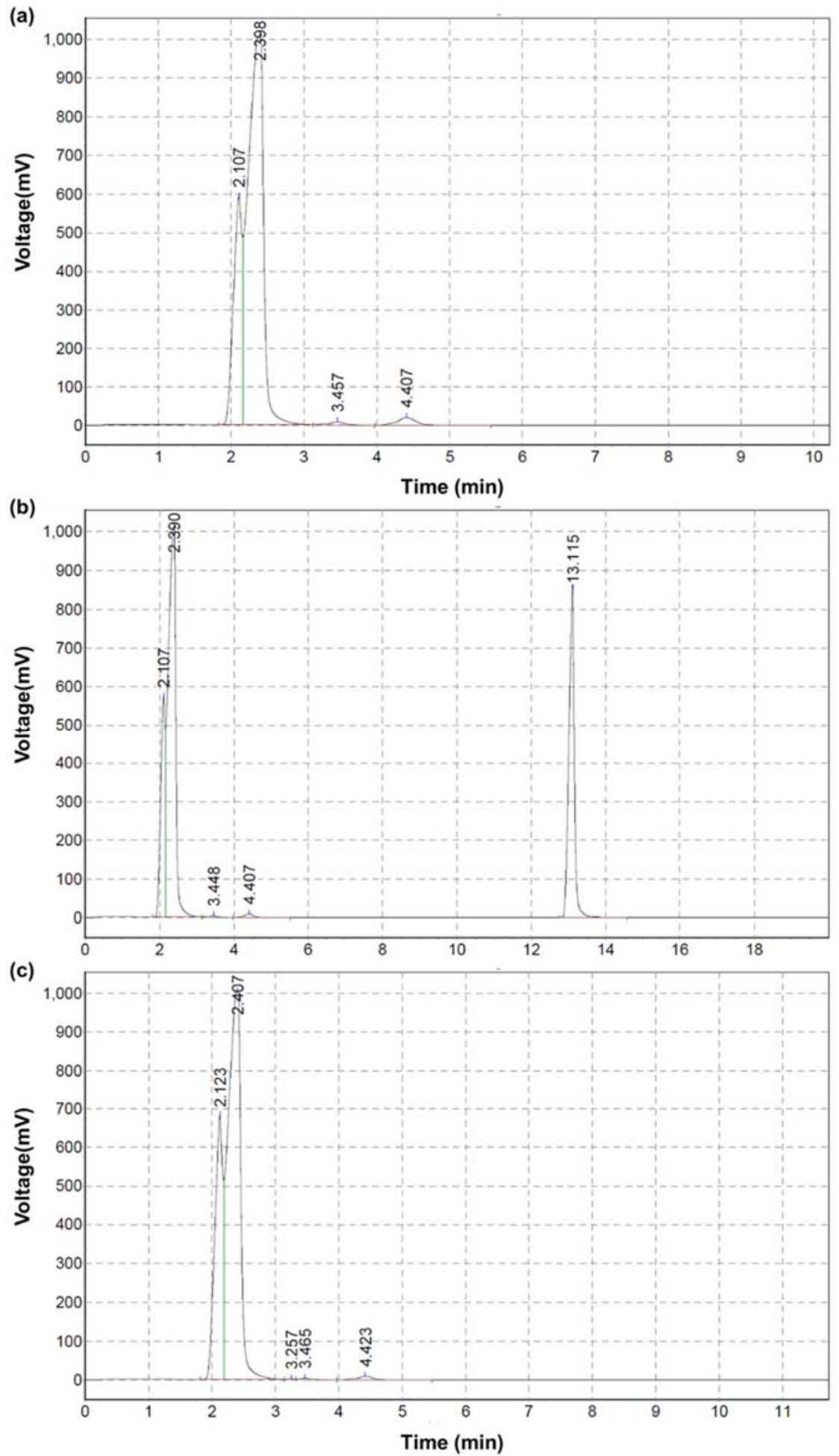

Figure S13. GC traces of generated $\mathrm{H}_{2}$ using (a) MG3-Au, (b) PVP-Au, and (c) dodecanethiol stabilized AuNPs as catalyst. The retention time for $\mathrm{H}_{2}$ is $2.4 \mathrm{~min}$. 
(a)

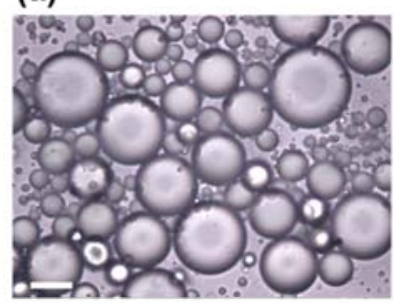

(d)

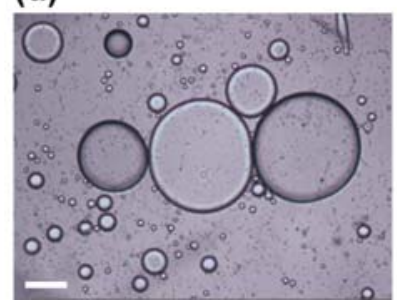

(b)

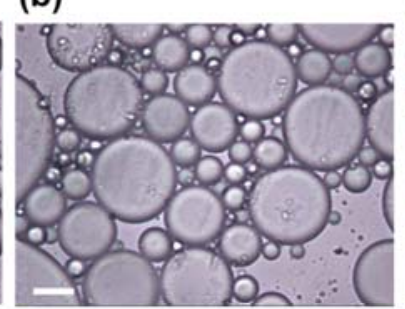

(e)

(c)

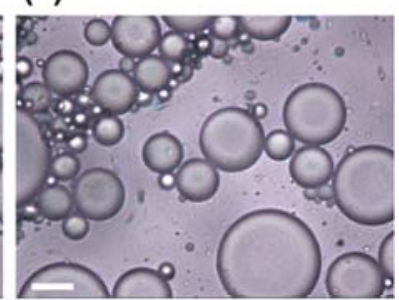

(f)
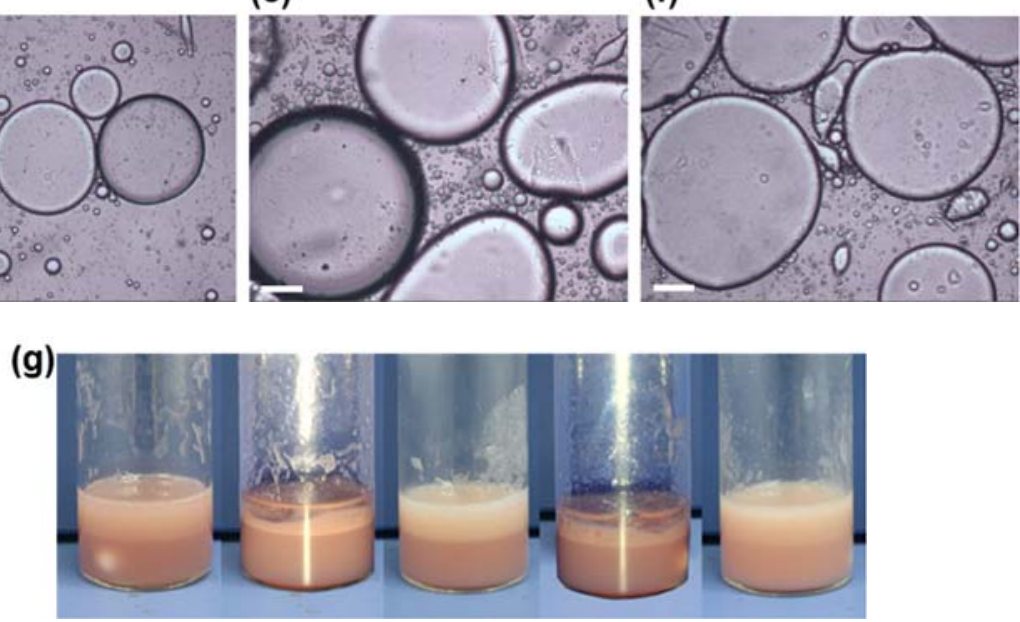

Figure S14. Microscope images and photos of MG3-Au stabilized Pickering emulsion. a-c) 5 min after homogenization of original state and $1^{\text {st }}, 2^{\text {nd }} \mathrm{CO}_{2} /$ vacuum boost (scale bar $\left.\left.=20 \mu \mathrm{m}\right) ; \mathrm{d}-\mathrm{f}\right) 1 \mathrm{hr}$ after homogenization of original state and $1^{\text {st }}, 2^{\text {nd }} \mathrm{CO}_{2} /$ vacuum boost (scale bar $\left.=50 \mu \mathrm{m}\right)$; g) photos of Pickering emulsion in original state and after two $\mathrm{CO}_{2} /$ vacuum treatments.

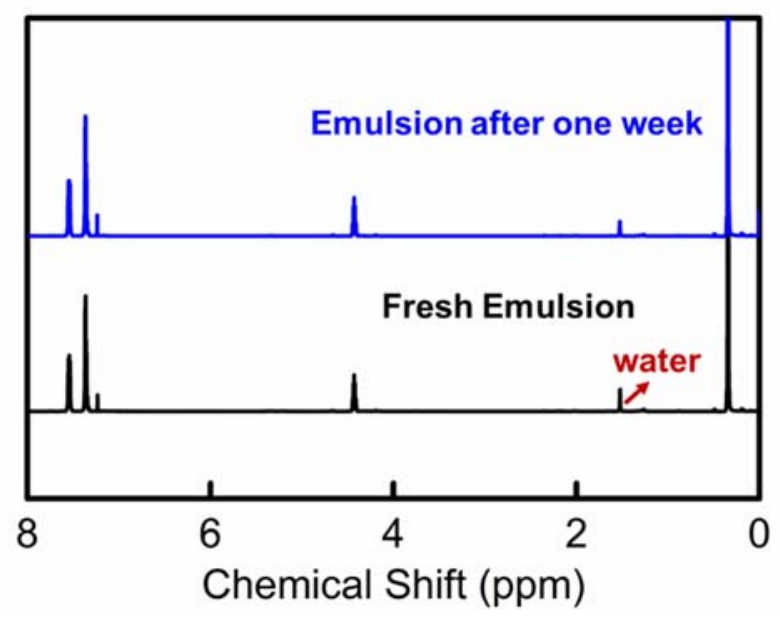

Figure S15. ${ }^{1} \mathrm{H}$ NMR spectra of dimethylphenylsilane emulsion comprising $2 \mathrm{~mL}$ of dimethylphenylsilane and $5 \mathrm{~mL}$ of $10 \mathrm{mg} / \mathrm{ml} \mathrm{MG3} \mathrm{solution} \mathrm{prior} \mathrm{and} \mathrm{subsequent} \mathrm{to} \mathrm{aging} \mathrm{for} \mathrm{one}$ week at room temperature. 
Table S1. Conversion in different systems with/without $\mathrm{CO}_{2}$ treatment

\begin{tabular}{|c|c|c|c|c|}
\hline & Substrate & $1 \mathrm{hr}$ & $\begin{array}{c}1^{\text {st }} \mathrm{CO}_{2} / \text { vacuum } \\
\text { cycle }\end{array}$ & $\begin{array}{c}2^{\text {nd }} \mathrm{CO}_{2} / \text { vacuum } \\
\text { cycle }\end{array}$ \\
\hline \multirow[t]{2}{*}{ MG } & & & $24.5 \%$ & $31.8 \%$ \\
\hline & $\mathrm{Et}_{3} \mathrm{SiH}$ & $12.8 \%$ & & \\
\hline $\mathrm{MG}-\mathrm{CO}_{2}$ & & & $28.7 \%$ & $53.6 \%$ \\
\hline \multirow[t]{2}{*}{ MG } & & & $53.4 \%$ & $93.4 \%$ \\
\hline & $\mathrm{PhMe}_{2} \mathrm{SiH}$ & $49.1 \%$ & & \\
\hline $\mathrm{MG}-\mathrm{CO}_{2}$ & & & $56.0 \%$ & $>99 \%$ \\
\hline
\end{tabular}

Reaction conditions: substrates $(2 \mathrm{~mL})$, water $(5 \mathrm{~mL}), 25^{\circ} \mathrm{C}$. Conversions were determined by ${ }^{1} \mathrm{H}$ NMR. MG samples both with and without $\mathrm{CO}_{2}$ are listed for comparison. Those with $-\mathrm{CO}_{2}$ stands for samples which were vacuumized/pressurized.

Table S2. Conversion of silane oxidation of MG-Au and recycles

\begin{tabular}{|c|c|c|c|c|}
\hline Entry & Substrate & MG-NP & Time(h) & Conv.(\%) \\
\hline 1 & & & 2 & $>99$ \\
\hline $1 \mathrm{a}$ & & & 2 & $>99$ \\
\hline & $\mathrm{Et}_{3} \mathrm{SiH}_{\mathrm{S}}$ & & & \\
\hline $1 b$ & & & 2 & $>99$ \\
\hline \multicolumn{5}{|c|}{$\mathrm{Au}$} \\
\hline $1 \mathrm{c}$ & & & 2 & $>99$ \\
\hline 2 & $\mathrm{Bu}_{3} \mathrm{SiH}$ & & 4 & $>99$ \\
\hline 3 & $\mathrm{PhMe}_{2} \mathrm{SiH}$ & & 4 & $>99$ \\
\hline
\end{tabular}

Reaction conditions: $\underline{\text { Substrates }}(10 \mathrm{mmol})$, water $(6 \mathrm{~mL})$, AuNPs/substrate $=0.24 \mathrm{~mol} \%, 25{ }^{\circ} \mathrm{C} .1 \mathrm{a}-\mathrm{c}$ are recycles of Run 1. Conversions were determined using ${ }^{1} \mathrm{H}$ NMR. 
Table S3. Conversion of olefin hydrogenation on MG-Pds and recycles

\begin{tabular}{|c|c|c|c|c|}
\hline Entry & Substrate & MG-NP & Time(h) & Conv.(\%) \\
\hline 1 & & & 4 & $>99$ \\
\hline \multirow[t]{2}{*}{$1 \mathrm{a}$} & & & 4 & $>99$ \\
\hline & BA & & & \\
\hline \multirow[t]{2}{*}{$1 b$} & & & 4 & $>99$ \\
\hline & & $\mathrm{Pd}$ & & \\
\hline $1 \mathrm{c}$ & & & 4 & $>99$ \\
\hline 2 & $\mathrm{EA}^{\mathrm{a}}$ & & 4 & $>99$ \\
\hline 3 & $\mathrm{St}^{\mathrm{b}}$ & & 4 & $>99$ \\
\hline
\end{tabular}

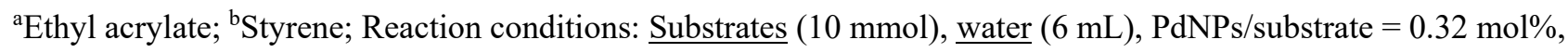
$25{ }^{\circ} \mathrm{C} .1 \mathrm{a}-\mathrm{c}$ are recycles of Run 1 . Conversion were determined using ${ }^{1} \mathrm{H}$ NMR. 


\section{REFERENCE}

(1) Melle, S., Lask, M., Fuller, G.G., Pickering emulsions with controllable stability, Langmuir, 2005, 21, 2158-2162, Pickering emulsions with controllable stability

(2) Levine, S., Bowen, B.D., Partridge, S.J., Stabilization of emulsions by fine particles I. Partitioning of particles between continuous phase and oil/water interface, Colloids and surfaces, 1989, 38, 325343, DOI: $10.1016 / 0166-6622(89) 80271-9$

(3) Destribats, M., Lapeyre, V., Wolfs, M., Sellier, E., Leal-Calderon, F., Ravaine, V., Schmitt, V., Soft microgels as Pickering emulsion stabilisers: role of particle deformability, Soft Matter, 2011, 7, 7689-7698, DOI: $10.1039 / \mathrm{C} 1 \mathrm{SM} 05240 \mathrm{C}$

(4) Geisel, K., Henzler, K., Guttmann, P., Richtering, W., New Insight into Microgel-Stabilized Emulsions Using Transmission X-ray Microscopy: Nonuniform Deformation and Arrangement of Microgels at Liquid Interfaces, Langmuir, 2015, 31, 83-89, DOI: 10.1021/1a503959n

(5) Richtering, W., Responsive emulsions stabilized by stimuli-sensitive microgels: emulsions with special non-Pickering properties, Langmuir, 2012, 28, 17218-17229, DOI: 10.1021/la302331s

(6) Teranishi, T., Kiyokawa, I., Miyake, M., Synthesis of monodisperse gold nanoparticles using linear polymers as protective agents, Adv. Mater., 1998, 10, 596-599, DOI: 10.1002/(SICI)15214095(199805)10:8<596::AID-ADMA596>3.0.CO;2-Y

(7) Brust, M., Walker, M., Bethell, D., Schiffrin, D.J., Whyman, R., Synthesis of thiol-derivatised gold nanoparticles in a two-phase liquid-liquid system, Journal of the Chemical Society, Chemical Communications, 1994, 801-802, DOI: 10.1039/C39940000801 